\title{
Comparative Assessment of Mould Growth Risk in Lightweight Insulating Assemblies Via Analysis of Hygrothermal Data and In Situ Evaluation
}

\author{
Inga Apine ${ }^{1 *}$, Mihails Birjukovs², Andris Jakovics ${ }^{2}$ \\ 1 University of Latvia, Plant Biology Laboratory, Kandavas street 2, Riga, Latvia \\ 2 University of Latvia, Laboratory for Mathematical Modelling of Technological and Environmental Processes, \\ Jelgavas street 3, Riga, Latvia \\ * Corresponding author's e-mail: inga.apine@lu.lv
}

\begin{abstract}
In order to assess the sustainability of buildings with different types of insulating assemblies in the Latvian climate, a long-term test building monitoring experiment has been underway since 2013. There are a total of five test buildings on site with roughly six years' worth of accumulated temperature and humidity readings in the key parts of assemblies. This study is meant to quantify the mould presence in building walls, floor and ceiling by performing laboratory tests, assessing the number of colony-forming units, and comparing the results with mould risk predictions due to the isopleth model developed by Sedlbauer, using both the hygrothermal data derived from the sensors within buildings and the output of numerical simulations in WUFI Pro 6.3, a commercial software package. The analysis indicated good agreement between the lab tests and mould risk assessment using the data sets from the sensors, validating the applicability of the Sedlbauer model to the Latvian climate, while the comparisons between the numerically obtained forecasts and experimental data revealed dissimilarities that are largely due to imprecisions in material models and initial conditions.
\end{abstract}

Keywords: building constructions, moisture, mould risk, WUFI, comparisons.

\section{INTRODUCTION}

Considering the whole life cycle of a building, the construction sector is the primary energy consumer in the modern world. One of the greatest challenges for the industry is to minimize its negative influence on the environment (Asadi et al. 2012, Santamouris 2016). In order to achieve this goal, starting from the design phase to the recycling or disposal phase, sustainability is the main keyword in the further development of the building industry (Pacheco-Torgal 2014, Czopka 2018). The progressively stricter requirements for the energy demand reduction and improvements in the energy efficiency lead to increasing the focus on the better insulated and more airtight building envelopes (including walls, floor, ceiling and roof) towards the implementation of nearly zero-energy buildings (NZEB) concept, which is included in the Directive 2010/31/EU of the European Union (Liobikiene and Mindaugas 2017, Dobbins et al. 2019).

Therefore, building design and construction must be oriented towards improving the performance of building envelopes by using standard, reliable solutions in combination with novel materials that must not only be effective, but also eco-friendly (Sinka et al. 2018, Boussaba et al. 2019). However, the increased airtightness and thicknesses of insulation in outer layers of building envelopes can lead to an overall increase in relative humidity $(\mathrm{RH})$ of building envelopes during the periods of lower exterior temperature, which would result in an increased risk of moisture-related damage, including mould growth (Gullbrekken et al. 2015, Mundt-Petersen and Harderup 2015). According to the RH requirements for different fungi to start growing and 
proliferate, fungi fall into several groups: xerophilic and xerotolerant $(<80 \% \mathrm{RH})$; mesophilic (80-90\% RH); hydrophilic ( $>90 \%)$ (Pasanen et al. 2000, Hess-Kosa 2010).

Despite the unremitting attention to the assessment of the hygrothermal performance of various envelope assemblies and boundary conditions associated with mould fungi growth, there are still gaps in knowledge concerning these issues (Mundt-Petersen and Harderup 2013, Vinha et al. 2018). Most of the studies on these problems have been carried out under laboratory conditions (Johansson et al. 2012, Laborel-Préneron et al. 2018), while there are very few detailed in situ investigations devoted especially to the microbial contamination, and efforts are usually limited to visual inspection (Mlakar and Štrancar 2013, Latif et al. 2014).

In order to avoid the moisture damage in buildings, a forecast of mould growth risk in buildings is very important already during the design phase, well before construction commences (Fedorik and Haapala 2018, Gradeci et al. 2018). To this end, various prediction models (Vereecken and Roels 2012, Gradeci et al. 2017) and numerical analysis tools (Fedorik and Haapala 2018, de Mello et al. 2019) were developed to the coupled transport of moisture and heat. One of the commonly used methods for mould growth risk assessment is the LIM (Lowest Isopleth for Mould) critical curve model developed by Sedlbauer (Krus et al. 2007). As for the simulation tools, WUFI ${ }^{\circledR}$ Pro is a rather popular and user-friendly software package for the hygrothermal simulations of building envelopes and can be used to solve the problems with symmetries that enable one-dimensional treatment of heat and moisture transport within building envelope cross-section. WUFI Pro also comes with an extensive material database (Künzel et al. 2012, Zirkelbach et al. 2016).

Although the WUFI numerical models have been validated for the walls in northern Europe area (Mundt-Petersen and Harderup 2013, Alev et al. 2014), the program has not been systematically tested for the Latvian climate, where the outdoor RH, temperature as well as wind speed differ from other European areas.

In order to comply with the NZEB standards, a long-term building performance monitoring project has been underway in Latvia since 2011 . Several small test buildings were built in the urban area of Riga (Latvia) using various local raw materials for different construction solutions. The main goal of the project is to evaluate the energy efficiency and sustainability of building constructions with similar U-values in the local climate (Dimdiņa et al. 2013, Ozolinsh and Jakovich 2013, Apine et al. 2015).

The objective of the present study was to evaluate the amount of mould fungi within the insulating assemblies (in wall, floor and ceiling) of test buildings by means of lab tests of material samples and compare the results against the mould growth risk predictions that are derived both from the experimental data obtained from sensors and the simulation results obtained using WUFI ${ }^{\circledR}$ Pro. This three-way analysis enables to verify the applicability of the standard LIM mould growth risk model to the local climate.

\section{MATERIALS AND METHODS}

\section{Test buildings}

In 2013, five test buildings with inner dimensions of $3 \times 3 \times 3 \mathrm{~m}$ and different wall insulating assemblies were completed. All wall assemblies have thermal transmittance of $\approx 0.16, \mathrm{~W} \cdot \mathrm{M}^{-2} \mathrm{~K}^{-1}$ and the floor and ceiling envelopes are identical across buildings. The indoor temperature $\sim 20^{\circ} \mathrm{C}$ and ventilation rate $0.45 \mathrm{~h}^{-1}$ is maintained within buildings. The buildings are equipped with a sensor and a data logger system that monitors the temperature, $\mathrm{RH}$, interior air velocity, solar radiation intensity, etc. - a typical layout of the sensor system within a building is shown in Fig. 1a. Detailed specifications for each building and sensor placement within are found in Jakovics et al. 2014. In this manuscript, the hygrothermal data insulation assemblies for three buildings were studied. Wall envelopes, codenamed LOG, PLY and AER, all have the following first three outer layers: Spruce, $40 \mathrm{~mm} \rightarrow$ Plywood, $6.5 \mathrm{~mm} \rightarrow$ Air gap, $30 \mathrm{~mm}$. Inner layer structure, exterior to interior, is as follows:

- LOG: Spruce, $200 \mathrm{~mm} \rightarrow$ Stone wool, $200 \mathrm{~mm} \rightarrow$ Vapor barrier, $<1 \mathrm{~mm} \rightarrow$ Spruce, $40 \mathrm{~mm}$ (Fig. 1b);

- PLY: Plywood, $20 \mathrm{~mm} \rightarrow$ Stone wool, $200 \mathrm{~mm}$ $\rightarrow$ Plywood, $20 \mathrm{~mm} \rightarrow$ Fibrolite, $75 \mathrm{~mm} \rightarrow$ Cement plaster, $20 \mathrm{~mm}$;

- AER: Stone wool, $30 \mathrm{~mm}$ Stone wool, $50 \mathrm{~mm}$ Lime-cement plaster, $15 \mathrm{~mm}$ Aerated concrete, $375 \mathrm{~mm} \rightarrow$ Lime-cement plaster, $15 \mathrm{~mm}$. 
- The ceiling and floor assemblies have the following layouts:

- Ceiling: Plywood, $12 \mathrm{~mm} \rightarrow$ Blowing birch wood fiber, $200 \mathrm{~mm} \rightarrow$ Plywood, $4 \mathrm{~mm} \rightarrow$ Stone wool, $50 \mathrm{~mm} \rightarrow$ Vapor barrier, $<1 \mathrm{~mm}$ $\rightarrow$ Plywood, 6.5 mm (Fig. 1b).

- Floor: Plywood, $21 \mathrm{~mm} \rightarrow$ Vapor barrier, $<$ $1 \mathrm{~mm} \rightarrow$ Stone wool, $200 \mathrm{~mm} \rightarrow$ Plywood, $21 \mathrm{~mm} \rightarrow$ Stone wool, $50 \mathrm{~mm} \rightarrow$ Vapor barrier, $<1 \mathrm{~mm} \rightarrow$ Plywood, $21 \mathrm{~mm}$.

The temperature and RH sensors were installed in key locations within wall, floor, and ceiling envelopes (see Fig. 1a and 1b). Additionally, monitoring weather conditions at the test site starting from April 2013 yielded the following data: outdoor temperature, $\mathrm{RH}$, solar radiation intensity, wind direction and speed. At the 5-year mark, the building envelopes were opened and the samples from the key locations were taken to the lab for the micro-organism content assessment.

\section{Collection of material samples for microbiological analysis}

Ceiling. In October 2017, the samples of blowing birch wood fibers were collected from
LOG (Fig. 1b). Three samples were taken from the top and three more from the bottom part of the insulation layer. Walls. In May 2018, the samples were collected from LOG and PLY outside surfaces of walls under ventilated facades, as well as exterior surfaces of stone wool slabs. No less than three samples were taken in each case. Floor. In August 2018, three samples were taken from exterior surface of the wool slab in the lower part of the AER floor envelope.

\section{Microbiological analysis}

In order to prepare a suspension for examination, $1 \mathrm{~g}$ of insulation material (stone wool or wood fiber) was homogenized in $100 \mathrm{ml}$ of sterilized distilled water. The surface samples were obtained using the swab method. In order to prepare a sample, a moistened cotton swab was used to wipe $10 \mathrm{~cm}^{2}$ of the wall surface. The swabs were placed in vials containing $1 \mathrm{ml}$ of sterile distilled water. At least three replications were prepared for each sample.

Afterwards, $100 \mu \mathrm{l}$ of obtained suspensions (from both insulation materials and surfaces of plywood and wood) were transferred onto Petri dishes with 2\% Malt Extract Agar and Dichloran

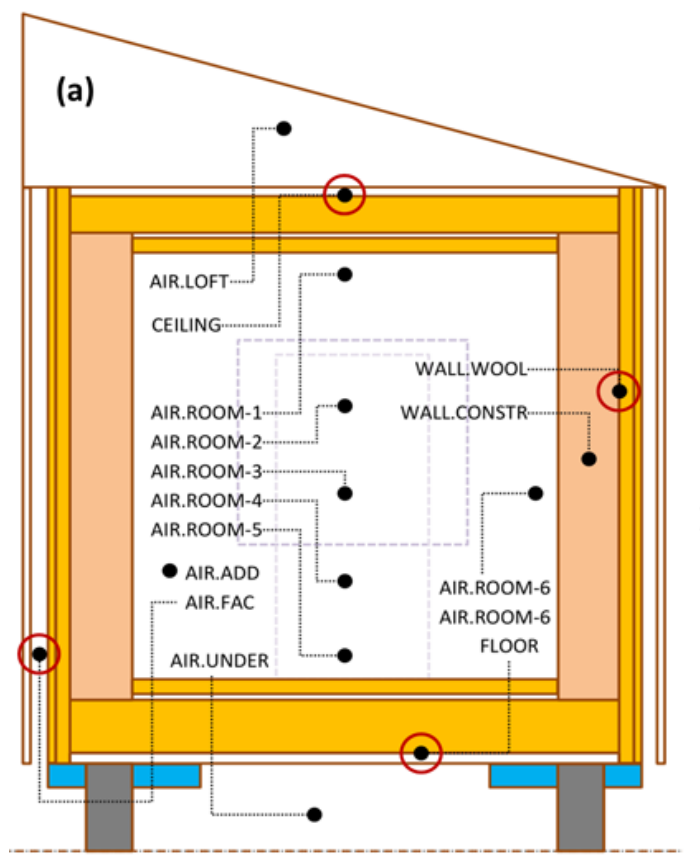

(b)
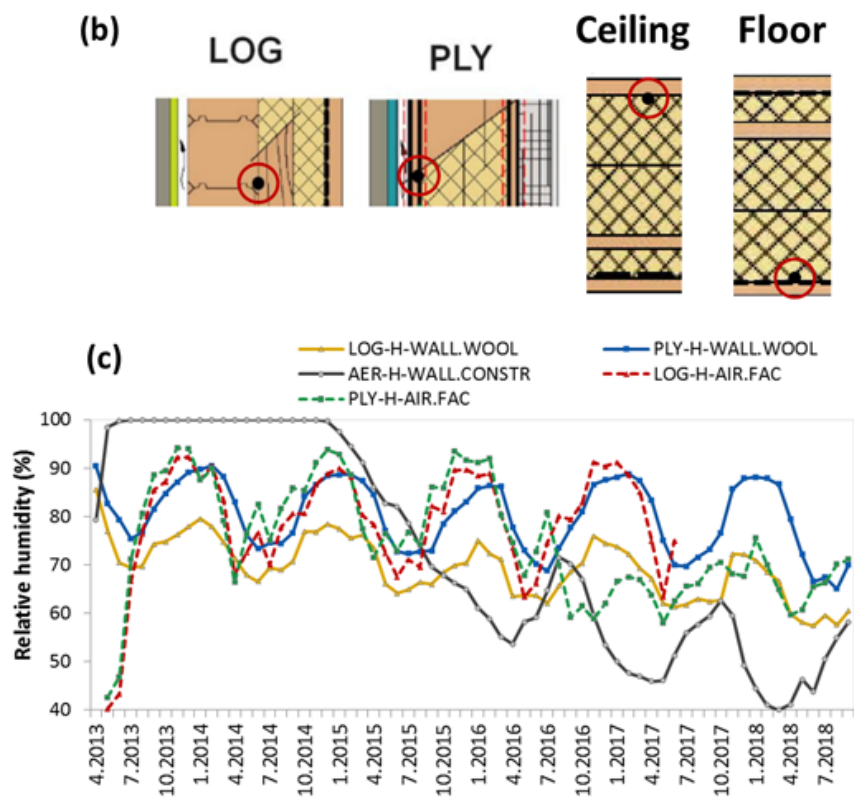

Figure 1. Location of the temperature and relative humidity sensors within a test building, marked with black dots (a); within wall, ceiling and floor insulating assemblies (b). Red circles mark the locations where the material samples were taken for lab analysis $(a, b)$. RH time series for key locations within buildings shown in (c). In (a), "ROOM" represents the sensors located within the building interior, "LOFT" is the attic sensor, "AIR. FAC" is the sensor within the air gap of the wall envelope, "WALL" and "FLOOR" are sensors embedded into walls and floor, respectively. 
$18 \%$ Glycerol Agar in at least four replications for each medium. Dichloran 18\% Glycerol Agar was used to isolate and identify xerophilic fungi (Penicillium, Aspargillus, Eurotium), while Malt Extract Agar allowed to resolve the mesophilic and hydrophilic moulds. When necessary, the solutions of the samples were additionally diluted from $10 \%$ of original concentration to $0.01 \%$. The plates with the samples were incubated at $20-23^{\circ} \mathrm{C}$ for $10-28$ days. Filamentous fungi were identified to the genera level using macro- and micro-morphological traits. The number of fungal propagules was expressed as colony-forming units (CFU) per gram of insulation material or per $\mathrm{cm}^{2}$ of material surface. The statistical differences among the samples were assessed using the Wilcoxon rank test.

\section{Mould growth prediction model}

In order to assess the mould fungi growth risk, Sedlbauer's Lowest Isopleth for Mould (LIM) model for mycelial growth was used (Krus et al. 2007). Four categories of substrates are defined to take into account not only RH and temperature, but also the effect of building materials as nutrient substrate: LIM 0 - optimal culture medium; LIM I - biologically degradable building materials (e.g. wall paper, martials made of biologically degradable raw resources, plaster); LIM II - biologically adverse materials (e.g. renderings, mineral building materials), LIM III - materials that are neither degradable nor contain nutrients (for this category is not given special isopleth curve).

The experimental data from sensors, temperature and RH time series, are converted to a series of sequential temperature/humidity value pairs and plotted against LIM curves ( 0 , I and II) - this allows one to both track the dynamics of mould growth risk and assess its severity.

\section{Numerical simulations}

The insulating assemblies were simulated in WUFI Pro 6.3, a commercial hygrothermal analysis software package. WUFI solves the coupled heat and moisture transport equations along with constitutive relations for the water content and water phase transitions. Given a sufficiently accurate history of the exterior climate and an adequate model for the interior climate, as well as correct material properties, it allows computing transient temperature and relative humidity fields within insulating assemblies. WUFI output, like sensor data, can be used to determine the mould growth risks throughout the simulated time interval of the experiment. A more detailed description of simulation methodology is given in Künzel (1995).

\section{RESULTS}

In the both PLY and LOG buildings the highest risk of mould growth is predicted in the outer part of insulation envelopes, wherein the sensors were placed at the stone wool layer boundary (Fig. 1b). For PLY, the sensor data indicates RH mostly above $70 \%$, but below $80 \%$ during the warm season over the first five years of monitoring (Fig. 1c). According to the Sedlbauer's model, such conditions should result in a moderate risk of mould growth. Moreover, in the initial stage of the experiment, the risk of fungal pollution was higher, but decreased slightly, then stabilized afterwards (Fig. 2). Although a visual inspection of stone wool slabs did not reveal any signs mould, the microbial analyses enable to detect the signs of quite significant fungal growth (Table 1). In PLY, not only xerophilic (Penicillium, Eurotium) fungi, but also a considerable concentration of mesophilic (Cladosporium, yeasts etc.) fungi were found. It should be noted that the construction of the test buildings was carried out during the autumn and winter, so the timber and insulation materials were exposed to damp air and moisture, which is likely to have increased RH considerably, promoting the mould growth.

However, the results of both the experiment and the simulation for PLY show that at the 5 year mark some risk of mould growth is present within the wall envelope. A visual inspection of the outer part of this assembly, the plywood surface within the air gap at the ventilated façade, was also conducted. It was found that the surface of plywood was mostly covered with black spots due to Cladosporium $\left(4038 \pm 1003 \mathrm{CFU} / \mathrm{cm}^{2}\right)$. This makes sense given that RH in the air gap consistently exceeded $70 \%$ during the warm seasons of the first five years of monitoring (Fig. 1c), demonstrating that the air change in the air gap was insufficient to prevent the mould growth and that an appropriate ventilation rate is a critical factor. (Hägerstedt and Arfvidsson 2010, Mundt-Petersen et al. 2013). At the end of 2017, the air flow rate through the ventilated facade was increased, resulting in the decline of RH. Further monitoring 

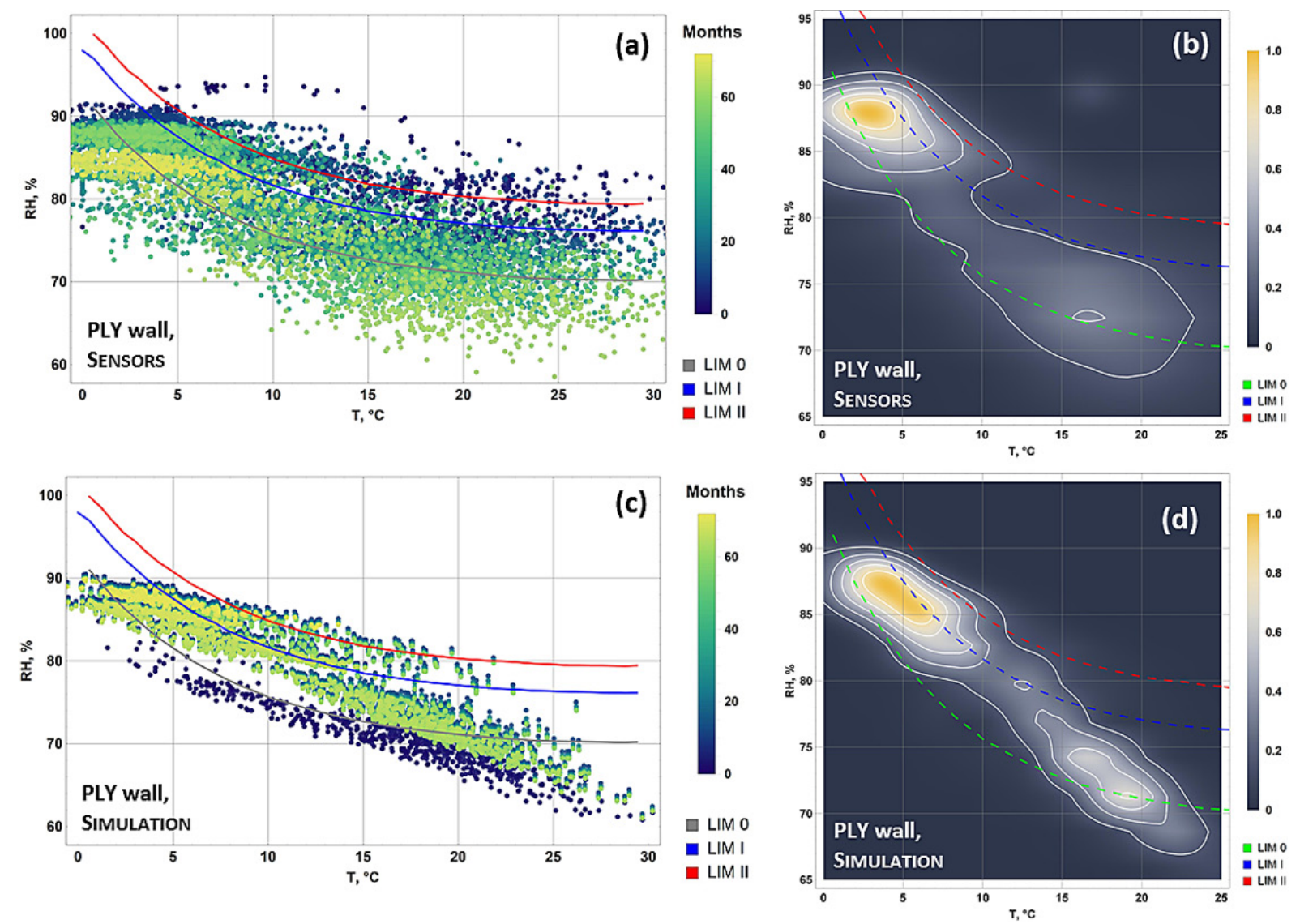

Figure 2. Mould growth risk assessment according to the Sedlbauer's model of mycelium growth (Krus et al. 2007) from April 3, 2013 to April 1, 2019 for the PLY wall at the location of the sensor placed within the stone wool layer. The mould growth risk forecasts due to the data from $(a, b)$ sensors and (c, d) WUFI are presented. In (a,c), the temperature/RH events are color coded in order of appearance, dark blue to yellow. In (b,d), color bars represent the relative density of events, higher values above the LIM curves indicating greater mould growth risk. White contours in $(b, d)$ are lines of constant event densit

Table 1. Concentration of culturable fungi in the stone wool samples from the insulation at the outer surfaces of LOG and PLY wall envelopes

\begin{tabular}{|c|c|c|c|c|c|c|c|}
\hline \multirow{2}{*}{\multicolumn{2}{|c|}{ Fungi }} & \multicolumn{6}{|c|}{ Concentration (CFU g $\left.{ }^{-1}\right)$} \\
\hline & & \multicolumn{3}{|c|}{ LOG } & \multicolumn{3}{|c|}{ PLY } \\
\hline \multirow{3}{*}{ Xerophilic } & Eurotium & 0 & \pm & 0 & 67 & \pm & 20 \\
\hline & Penicillium & 188 & \pm & 31 & 550 & \pm & 101 \\
\hline & Total & 188 & \pm & 31 & 617 & \pm & 121 \\
\hline \multirow{4}{*}{ Mesophilic } & Cladosporium & 150 & \pm & 29 & 12350 & \pm & 1487 \\
\hline & Yeasts & 125 & \pm & 48 & 200 & \pm & 67 \\
\hline & Others* & 175 & \pm & 66 & 200 & \pm & 38 \\
\hline & Total & 450 & \pm & 143 & 12750 & \pm & 1592 \\
\hline \multicolumn{2}{|c|}{ TOTAL } & 638 & \pm & 174 & 13400 & \pm & 1709 \\
\hline
\end{tabular}

is necessary to ascertain that this will reduce the mould growth risk.

In the LOG wall assembly, the sensor data has revealed that the hygrothermal conditions were favorable for the mould growth, with many events above LIM I and LIM II curves, mostly during a short time period after the construction was finished (Fig. 3). In the examined samples, the fungal contamination could not be identified visually. However, lab tests allowed to detect a minor amount of fungi, mostly Penicillium, Cladosporium and yeasts (Tab. 1) with the total 

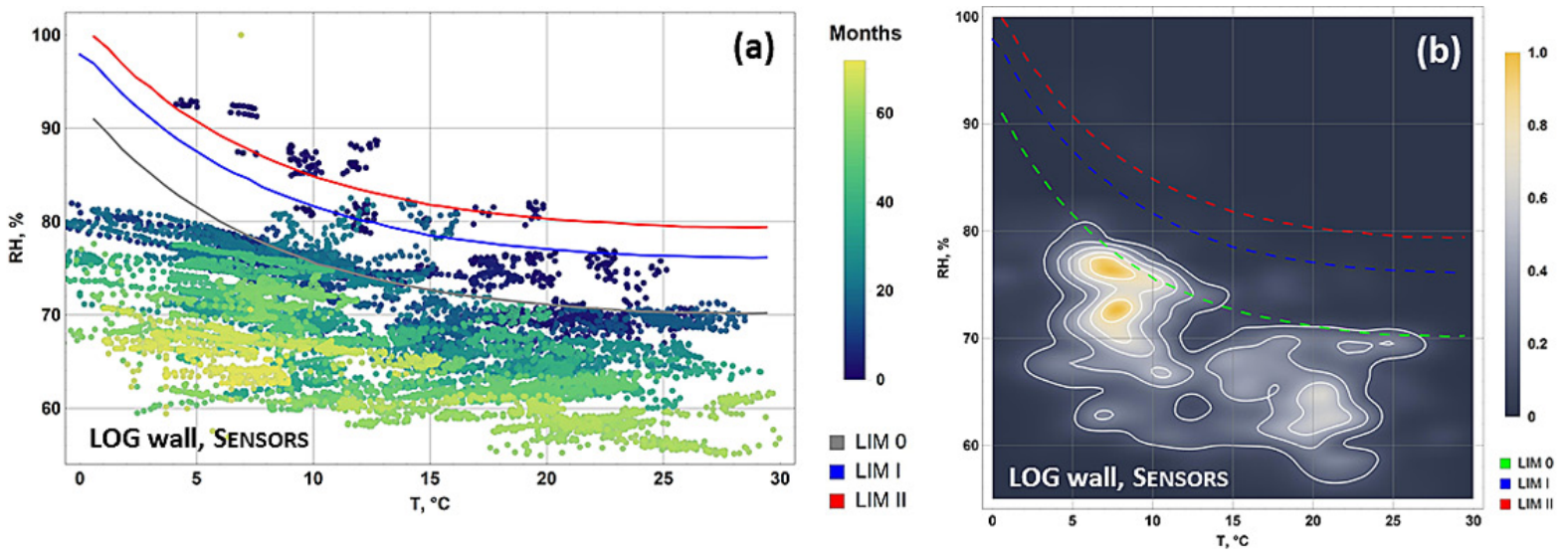

Figure 3. Mould growth risk assessment according to the Sedlbauer's model from April 3, 2013 to April 1, 2019 for the LOG wall assembly at the location of the sensor placed within the stone wool layer

amount significantly lower (statistical p-value $\mathrm{p}<0.001)$ than in PLY. As in the PLY case, the risk of mould growth has been decreased over the period of five years. The timber behind the ventilation façade was partially covered with black spots of Cladosporium fungi, which is, again, due to insufficient ventilation. Here, the RH trends were similar to those observed for PLY (Fig. 1c).

Unfortunately, the material model for the LOG wall assembly has not been established yet. Therefore, the LOG numerical results are not presented here.

The sensor data for LOG ceiling envelope shows that during the first years of monitoring, the RH and temperature conditions supported the fungal growth within blowing birch wood fiber - there is a high density of the RH/temperature events even above LIM II, though the highest density is below LIM 0 (Fig. 4). Over 5 years, the mould growth risk has declined. The microbiological analysis confirmed the presence of fungal pollution. During a visual inspection, the insulation wood fiber under the cover plywood appeared coated with a thin film of mould. The samples from wood fiber at the sensor location
(Fig. 1b) had a considerable concentration of xerophilic species, i.e. Penicillium, Aspargillus and Eurotium, and even higher concentration $(\mathrm{p}<0.001$ was found in the samples taken from the lower part of ceiling insulation (Table 2).

As seen in Fig.4, the simulation results yield a different pattern for the mould growth. The risk of fungi proliferation was significantly lower at the beginning of the monitoring period. However, note that the projected mould risk increased rapidly and the RH/temperature event density was greatest in the area between LIM 0 and LIM I, with a fraction of time spent above LIM I, indicating minor mould growth risk within wood fiber insulation. As in the PLY wall case, the predicted RH values increase over time, indicating that the envelope materials accumulate moisture. To summarize, while the simulation results revealed a minor mould growth risk with a tendency to increase over time, the experimental data suggests the opposite dynamics. Further monitoring of the ceiling envelope, as well as improvement of material models and more adequate initial conditions for simulations is necessary to understand the factors that lead to discrepancies.

Table 2. Concentration of culturable fungi in the samples of blowing birch wood fiber from LOG ceiling insulation layer

\begin{tabular}{|c|c|c|c|c|c|c|c|}
\hline \multirow{2}{*}{\multicolumn{2}{|c|}{ Fungi }} & \multicolumn{6}{|c|}{ Concentration (CFU g $\left.{ }^{-1}\right)$} \\
\hline & & \multicolumn{3}{|c|}{ Top } & \multicolumn{3}{|c|}{ Bottom } \\
\hline \multirow{4}{*}{ Xerophilic } & Aspargillus & 330493 & \pm & 30221 & 12167042 & \pm & 2263724 \\
\hline & Eurotium & 1544 & \pm & 657 & 33958 & \pm & 4104 \\
\hline & Penicillium & 7464845 & \pm & 624884 & 358602013 & \pm & 13652617 \\
\hline & Total & 7796883 & \pm & 655762 & 370803014 & \pm & 15920445 \\
\hline \multirow[t]{2}{*}{ Mesophilic } & Different* & 0 & \pm & 0 & 525 & \pm & 361 \\
\hline & Total & 0 & \pm & 0 & 525 & \pm & 361 \\
\hline \multicolumn{2}{|c|}{ TOTAL } & 7796883 & \pm & 655762 & 370803014 & \pm & 15920445 \\
\hline
\end{tabular}



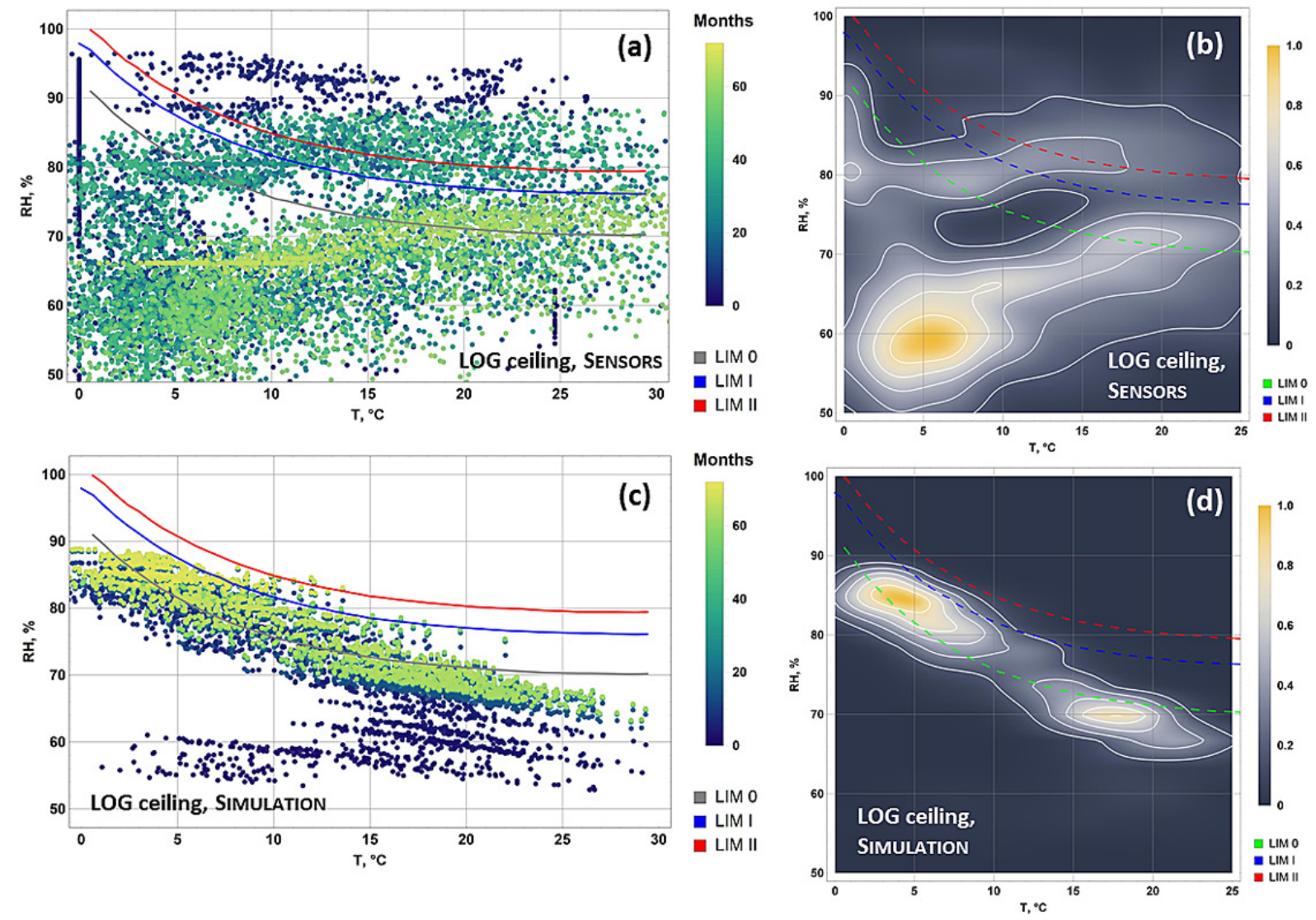

Figure 4. Mould growth risk assessment according to the Sedlbauer's model from April 3, 2013 to April 1, 2019 for LOG ceiling at the location of the sensor placed on top of the layer of blowing birch wood fiber using the data from $(a, b)$ sensors and (c, d) WUFI

The floor insulating assembly was identical for all buildings. However, the initial conditions may have been dissimilar due to the exposure to moist air during construction. Moreover, a critical error was made during construction - an additional vapor barrier was included at the bottom of the envelope, below the insulation layers, therefore making the assembly vapor-tight. Combined with high initial RH due to the exposure to ambient air, the mould growth hazard was, as indicated by analysis of the AER building, the highest among all assemblies and persisted throughout the monitoring period, as seen from both sensor and simulation output shown in Figure 5.

Note that both experiments and simulations produce the $\mathrm{RH} /$ temperature events concentrated well above LIM II curves. The mould growth was more pronounced during the cold seasons, where the temperature ranged from $0^{\circ} \mathrm{C}$ to $5^{\circ} \mathrm{C}$. The laboratory tests confirmed the results obtained from sensors and simulations. Although a visual inspection did not indicate any mould growth, the sample analysis revealed a rather high concentration of xerophilic (Penicillium, Eurotium) and mesophilic (Cladosporium, Epicoccum, yeasts etc.) fungi (Table 3 ).

On the numerical simulations side, a much worse situation in terms of fungi proliferation is predicted during warmer periods within $10^{\circ} \mathrm{C}$ to $17^{\circ} \mathrm{C}$ range. This is likely due to overestimated vapor diffusion resistance of the vapor barrier at the bottom of the envelope. The building maintenance logs indicate that floor vapor barriers may have been damaged, which could result in partial perforation and translate to lower vapor diffusion resistance coefficients. A numerical sensitivity analysis will be performed for the floor insulating assembly to address this issue.

\section{CONCLUSIONS}

The results of this study clearly show that Sedlbauer's LIM curve model for mould growth risk assessment can be safely and successfully applied in the Latvian climate. Clearly, even longer monitoring periods are necessary to evaluate the sustainability of different insulation 

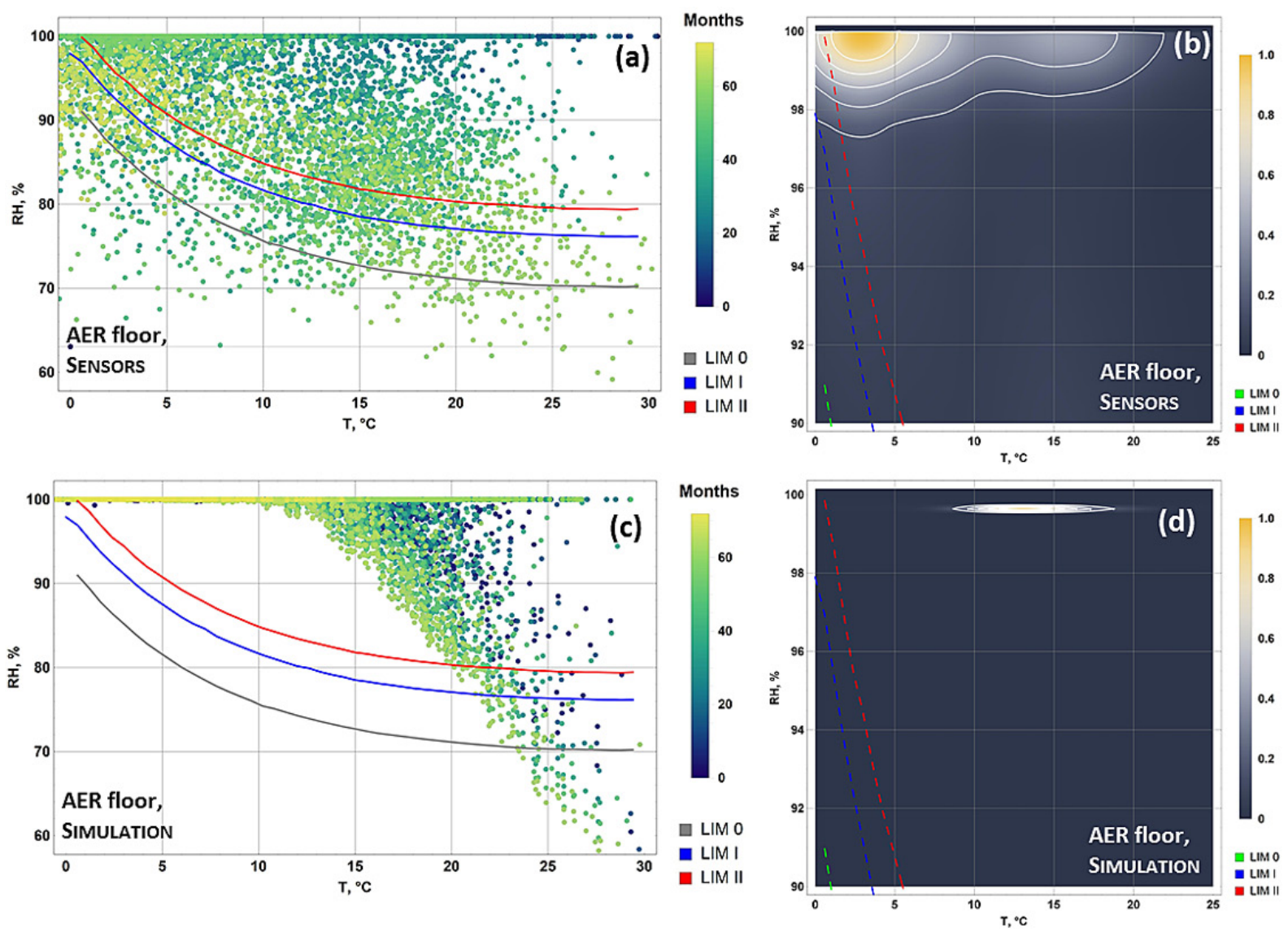

Figure 5. Mould growth risk assessment according to the Sedlbauer's model from April 3, 2013 to April 1, 2019 for AER floor at the location of the sensor placed at the lower layer of stone wool using data from (a, b) sensors and $(c, d)$ WUFI

solutions, which is the experiment currently continues, likely for several more years. Comparing the simulated mould growth risk dynamics against the sensor data and lab tests, some dissimilarities were found. These are largely due to imprecisions in materials models (i.e. sorption

Table 3. Concentration of culturable fungi within the samples of stone wool insulation from the AER floor envelope

\begin{tabular}{|c|c|c|c|c|}
\hline \multicolumn{2}{|c|}{ Fungi } & \multicolumn{3}{|c|}{ Concentration $\left(\mathrm{CFU} \mathrm{g^{-1 } )}\right.$} \\
\hline \multirow{3}{*}{ Xerophilic } & Eurotium & 250 & \pm & 144 \\
\hline & Penicillium & 98383 & \pm & 26443 \\
\hline & Total & 98633 & \pm & 26587 \\
\hline \multirow{6}{*}{ Mesophilic } & Alternaria & 333 & \pm & 110 \\
\hline & Cladosporium & 9333 & \pm & 3112 \\
\hline & Epicoccum & 583 & \pm & 220 \\
\hline & Yeasts & 5069 & \pm & 2015 \\
\hline & Others* & 42 & \pm & 21 \\
\hline & Total & 15361 & \pm & 5478 \\
\hline \multicolumn{2}{|c|}{ TOTAL } & 113994 & \pm & 32065 \\
\hline
\end{tabular}

* Fungi identified only once or twice, such as Chrysonilia, Geotrychum, Isariella, Paecilomuces, Phoma, Phomopsis etc. curves) and initial conditions (actual versus input inferred from sensor data) for RH within assemblies. However, in the case of the PLY wall assembly, the numerical model correlates with the lab test results quite well. The results are currently being used to validate the material models for WUFI Pro 6.3, which can then be used by other researchers, improving WUFI as a valuable tool that can be used to avoid critical errors during the design phase of building construction and to ensure that the insulating assemblies perform as intended over the course of building operational lifetime.

\section{Acknowledgments}

This study was conducted with the financial support of European Regional Development Fund, as part of the project "Development, optimization and sustainability evaluation of smart solutions for nearly zero energy buildings in real climate conditions" (1.1.1.1/16/A/192). The authors are very grateful to Dr. Vizma Nikolajeva for crucial advice and assistance in fungi identification. 


\section{REFERENCES}

1. Alev U., Uus A., Teder M., Miljan M.J., Kalamees T. 2014. Air leakage and hygrothermal performance of an internal insulated log house. The 10th Nordic Symposium on Building Physics.Lund, Sweden, 55-62

2. Apine I., Orola L., Jakovics A. 2015. Effect of building envelope materials on indoor air quality in low energy test houses. International Journal of Environmental Science and Development, 6(12), 952-957.

3. Asadi E., da Silva M.G., Antunes C.H., Dias L. 2012. Multi-objective optimization for building retrofit strategies: a model and an application. Energy and Buildings, 44, 81-87

4. Boussaba L., Makhlouf S., Foufa A., Lefebvre G., Royon L. 2019. Vegetable fat: A low-cost bio-based phase change material for thermal energy storage in buildings. Journal of Building Engineering, 21, 222-229.

5. Czopka P. 2018. Sustainable materials in ecological buildings. Economic and Environmental Studies, 18(45), 93-102.

6. de Mello L.A., Moura L.M., Mendes N. 2019. A model for assessment of heat and moisture transfer through hollow porous buildings elements. Case Studies in Thermal Engineering, 14, 100446., 13 p.

7. Dimdiņa I., Jakovičs A., Gendelis S., Kḷaviņš J. 2013. Testing of energy-efficient building envelope materials in natural conditions. Proc. Int. Conf. on World Sustainable Energy Days, Wels, Austria, 1-5.

8. Dobbins A., Nerini F.F., Deane P., Pye S. 2019. Strengthening the EU response to energy poverty. Nature Energy, 4(1), 2-5.

9. Fedorik F., Haapala A. 2018. Numerical estimation of mould growth on common single-family house building envelopes in boreal conditions. European Journal of Environmental and Civil Engineering, 22(10), 1196-1211.

10. Gradeci K., Berardi U., Time B., Köhler J. 2018. Evaluating highly insulated walls to withstand biodeterioration: A probabilistic-based methodology. Energy and Buildings, 177, 112-124.

11. Gradeci K., Labonnote N., Time B., Köhler J. 2017. Mould growth criteria and design avoidance approaches in wood-based materials - a systematic review. Construction and building materials, 150, 77-88.

12. Gullbrekken L., Geving S., Time B., Andresen I. 2015. Moisture conditions in passive house wall constructions. Energy Procedia, 78, 219-224.

13. Hägerstedt S.O., Arfvidsson J. 2010. Comparison of field measurements and calculations of relative humidity and temperature in wood framed walls. Proc. 15th International Meeting of Thermophysical Society, Valtice, Czech Republic, 93-101.

14. Hess-Kosa K. 2010. Indoor air quality: Sampling methodologies, CRC Press, Florida.

15. Jakovich A., Gendelis S., Ozolins A., Sakipova S.E. 2014. Energy efficiency and sustainability of low energy houses in Latvian climate conditions. Proc. Int. Conf. Energy, Environment, Development and Economics, Santorini, Greece, 109-114.

16. Johansson P.; Ekstrand-Tobin A.; Svensson T., Bok G. 2012. Laboratory study to determine the critical moisture level for mould growth on building materials. International Biodeterioration and Biodegradation, 73, 23-32.

17. Krus M., Kilian R., Sedlbauer K. 2007. Mould growth prediction by computational simulation on historic buildings. In: Padfield T., Borchersen K. (eds), In Museum Microclimates. Copenhagen, Denmark, The National Museum of Denmark.

18. Künzel H.M. 1995. Simultaneous heat and moisture transport in building components. One-and twodimensional calculation using simple parameters. Ph.D. Thesis, Frauhofer IRB-Verlag Stuttgart.

19. Künzel H.M., Zirkelbach D., Schafaczek B. 2012. Modelling the Effect of Air Leakage in Hygrothermal Envelope Simulation National Institute of Building Science. Proc. BEST3 Conference Atlanta, $13 \mathrm{p}$.

20. Laborel-Préneron A., Ouédraogo K., Simons A., Labat M., Bertron A., Magniont C. et al. 2018. Laboratory test to assess sensitivity of bio-based earth materials to fungal growth. Building and Environment, 142, 11-21.

21. Latif E., Ciupala M.A., Wijeyesekera D.C. 2014. The comparative in situ hygrothermal performance of Hemp and Stone Wool insulations in vapour open timber frame wall panels. Construction and Building Materials, 73, 205-213.

22. Liobikiene G., Mindaugas B. 2017. The European Union possibilities to achieve targets of Europe 2020 and Paris agreement climate policy. Renewable Energy, 106, 298-309.

23. Mlakar J., Štrancar J. 2013. Temperature and humidity profiles in passive-house building blocks. Building and Environment, 60, 185-193.

24. Mundt-Petersen O., Harderup L.E., Arfvidsson J. 2013. Important factors affecting the risk of mold growth in well - Insulated wood frame walls in northern European climates. Proc. of the Thermal Performance of the Exterior Envelopes of Whole Buildings XII, $13 \mathrm{p}$.

25. Mundt-Petersen S.O., Harderup L. E. 2015. Predicting hygrothermal performance in cold roofs using a 1D transient heat and moisture calculation tool. Building and Environment, 90, 215-231.

26. Mundt-Petersen S.O., Harderup L.E. 2013. Validation of a one-dimensional transient heat and moisture 
calculation tool under real conditions. Proc. of the Thermal Performance of the Exterior Envelopes of Whole Buildings XII-International Conference Florida USA, $12 \mathrm{p}$.

27. Ozolinsh A., Jakovich A. 2013. Risks of condensate formation and mould growth in buildings under Latvian climate conditions. Latvian Journal of Physics and Technical Sciences, 5, 1-11.

28. Pacheco-Torgal F. 2014. Eco-efficient construction and building materials research under the EU Framework Programme Horizon 2020. Construction and Building Materials, 51, 151-62.

29. Pasanen A.L, Rautiala S., Kasanen J.P., Raunio P., Rantamaki J., Kalliokoski P. 2000. The relationship between measured moisture conditions and fungal concentrations in water-damaged building materials. Indoor Air, 11(2), 111-120.

30. Santamouris M. 2016. Innovating to zero the building sector in Europe: Minimising the energy consumption, eradication of the energy poverty and mitigating the local climate change. Solar Energy, 128, 61-94.

31. Sinka M., Bajare D., Gendelis S., Jakovics A. 2018. In-situ measurements of hemp-lime insulation materials for energy efficiency improvement. Energy Procedia, 147, 242-248.

32. Vereecken E., Roels S. 2012. Review of mould prediction models and their influence on mould risk evaluation. Building and Environment, 51, 296-310.

33. Vinha J., Salminen M., Salminen K., Kalamees T., Kurnitski J., Kiviste M. 2018. Internal moisture excess of residential buildings in Finland. Journal of Building Physics, 42(3), 239-258.

34. Zirkelbach D., Tanaka E. 2016. Local Climate Models for Hygrothermal Building Component Simulations. Proc. of the CESBP Central European Symposium on Building Physics and BauSIM, 149-155. 